\title{
Wet processing of Recent calcareous foraminifera: methods for preventing dissolution.
}

\author{
C. A. MAYBURY \\ Institute of Earth Studies \\ University College of Wales \\ Aberysturyth \\ Dyfed, $S Y 233 D B$ \\ U.K.
}

\author{
I. AP GWYNN \\ Department of Biological Sciences \\ University College of Wales \\ Aberystwyth \\ Dyfed, SY23 3DA \\ U.K.
}

\begin{abstract}
Two methods for the wet processing of Recent foraminifera are described: one utilizing glutaraldehyde, the other formaldehyde. Both minimize the effects of dissolution on the tests of Recent calcareous foraminifera. J. Micropalaeontol., 12 (1): 67-69, August 1993.
\end{abstract}

\section{INTRODUCTION}

Encouraged by the large response to Maybury et al.'s note on mountants for 'live' foraminifera (Micropalaeontology Notebook Vol. 9(2) p.172 and Vol. 10(1) p.16), we present another major problem pertinent to the study of Recent foraminifera in the hope of generating further comments and ideas. This is the problem of dissolution of calcareous specimens (Fig.1). It pertains to Recent material that needs to be wet sorted and contains calcareous specimens which cannot be picked and dry mounted soon after collection. We refer particularly to material collected at sea, where there may be an interval of several months before it arrives in the laboratory. There are 4 stages generally involved in the wet processing of Recent foraminifera subsequent to their collection: fixation, preservation, staining and sorting. Dissolution of calcareous tests and also of calcareous cements in agglutinating foraminifera may occur at any stage during processing if the $\mathrm{pH}$ of the containing medium is not maintained above 8.6. Hodgkinson (1991), quoting from Hay (1977) and Lewin (1961), notes that a pH between 8.6 and 9.4 is considered safe. The Hay and Lewin references, however, are referring to calcareous nannofossils and siliceous diatoms respectively and hence this $\mathrm{pH}$ range might be considered a little high for foraminifera. Pfannkuche \& Thiel (1988) quote a minimum $\mathrm{pH}$ of 8.2 for the prevention of calcium carbonate dissolution. Our observations, however, do not reveal any deterioration in the specimens when they are stored, for example, at $\mathrm{pH}$ 9.22. All $\mathrm{pH}$ values quoted herein relate to freshly made solutions and we have also indicated the degree of purity of the chemicals used, e.g., electron microscopy quality, analytical reagent quality and laboratory reagent quality. In all cases we would always recommend using the purest chemicals available. Below we outline 2 standard methods which we have modified slightly to minimize the dissolution of calcareous tests.

\section{METHOD I: GLUTARALDEHYDE}

The penetration of glutaraldehyde into most soft tissue is about $0.5 \mathrm{~mm}$ and so it should be a suitable fixative for the majority of foraminifera (up to $1 \mathrm{~mm}$ in maximum dimension). This method is recommended for material that is to undergo any form of detailed analysis such as critical point drying, thin sectioning, isotope analysis, mass spectrometry etc. It must be emphasized, however, that baseline readings of all the containing media should be recorded, e.g., in isotope analysis and mass spectrometry and adjustments made to allow for the chemicals that these media contain. The BDH Laboratory Supplies Catalogue from Merck Ltd provides useful assays of these chemicals. Once treated the specimens can then be stored in a suitable buffer and their preservation is permanent.

\section{Fixation}

Place marine sediment sample in plastic container with a screw top and add $2 \%$ glutaraldehyde. Use an equal volume of glutaraldehyde to the sediment volume. [To make a stock solution of $2 \%$ glutaraldehyde take $8 \mathrm{ml}$ electron microscopy quality $25 \%$ glutaraldehyde and add to $92 \mathrm{ml}$ filtered seawater. Dissolve $1 \mathrm{~g}$ analytical reagent quality calcium chloride 6-hydrate in this mixture. Filter the solution to remove any undissolved crystalline material. The $\mathrm{pH}$ of this solution is 6.75 at $20^{\circ} \mathrm{C}$. Gently agitate the closed plastic container to ensure all sediment particles and organisms are thoroughly saturated. Do not shake the 
container violently or specimens may be broken. After 1 hour filter the contents of the container very gently under reduced pressure using standard palynological techniques (Batten \& Morrison, 1983 p.38). It is important to remove the glutaraldehyde solution from the sediments or polymerization can occur; but the sediments should remain wet at all times. For this purpose wash the sediments continuously with filtered seawater contained within a wash bottle. The fixative's low $\mathrm{pH}$ does not appear to cause the dissolution of calcareous tests; probably because they are not exposed to it for long.

\section{Preservation}

When the glutaraldehyde has been washed from the sediments, add a solution of sodium cacodylate and agitate gently as before. [To make a stock solution of sodium cacodylate, dissolve $21 \mathrm{~g}$ laboratory reagent quality sodium cacodylate in 11 filtered seawater. Filter the mixture to remove any undissolved chemicals. The $\mathrm{pH}$ of this solution is 8.93 at $20^{\circ} \mathrm{C}$. Specimens may be stored in this medium indefinitely. Use equal sediment and preservative volumes.

\section{Staining}

Rinse sample on appropriate sieve with a wash bottle containing a solution of $4 \mathrm{~g}$ analytical reagent quality ammonium hydrogen carbonate dissolved in 11 distilled water ( $\mathrm{pH} 8.60$ at $20^{\circ} \mathrm{C}$ ). Stain overnight by immersing sieve in a solution made from $1 \mathrm{~g}$ rose bengal and $4 \mathrm{~g}$ analytical reagent quality ammonium hydrogen carbonate in 11 distilled water.

Sorting

Wash excess stain off the sample with a wash bottle containing ammonium hydrogen carbonate in distilled water (quantities as above). Use this solution as the sorting medium.

\section{Specific hazards}

Glutaraldehyde should always be handled in a fume cupboard, using nitrile gloves and safety goggles. It is harmful by ingestion and inhalation and extremely irritating to the eyes. Prolonged skin contact can cause dermatitis and sensitisation. Further toxicity data, first aid, spillage, disposal, storage and handling advice and details on reactive hazards and protective measures can be obtained from the BDH Hazard Data Sheets from Merck Ltd. 'Spills can be neutralized by solid glycine (chromatographically homogeneous). Alternatively, a bottle containing $1 \mathrm{M}$ glycine can be kept on hand to spray on the spill. One volume of this glycine renders an equivalent volume of $50 \%$ (or less) glutaraldehyde non-volatile' Hayat, 1989.

Sodium Cacodylate 'contains approximately 30\% arsenic by weight. This arsenic presents hazards of ingestion, skin contact and inhalation, nerve paralysis and cancer of the liver, skin and lung. Symptoms include nervousness, thirst, vomiting, diarrhoea, cyanosis and collapse. It is a cumulative poison and inhalation of small concentrations over a long period will cause poisoning. Eyes should be
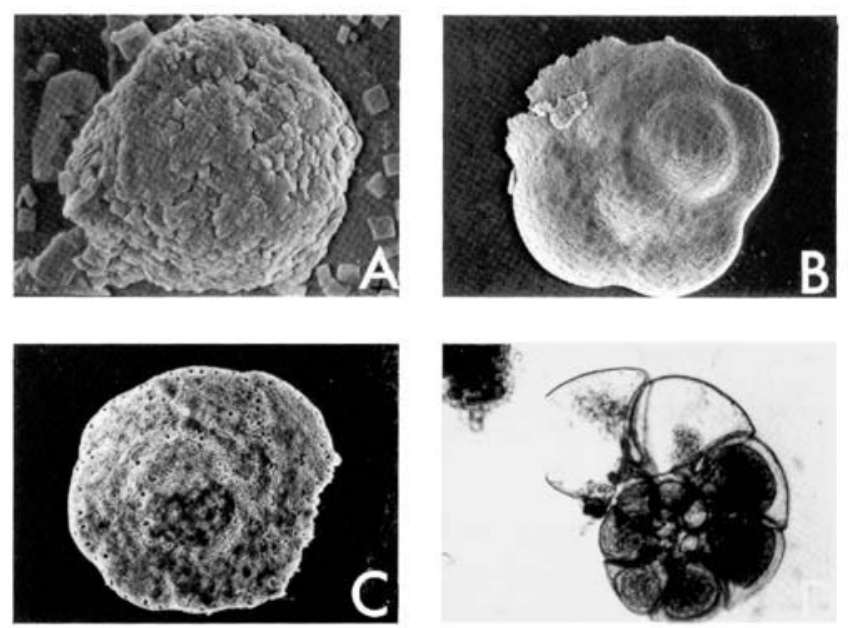

Fig 1.

a. Halite crystals on poorly preserved saccamminid? Scale bar $25 \mu \mathrm{m}$. b. Dissolution mosaic on surface of rotaliid. Scale bar $25 \mu \mathrm{m}$

c. Severe dissolution of rotaliid rendering identification impossible. Scale bar $50 \mu \mathrm{m}$.

d. Complete dissolution of test, only organic wall and protoplasm remaining. The specimen is possibly Nonion. Scale bar $50 \mu \mathrm{m}$.

Figs 1a-c, scanning electron micrographs; Fig. 1d, light micrograph. The specimens illustrated in Figs $1 \mathrm{~b}$-d have been fixed and preserved in $4 \%$ formaldehyde in filtered seawater buffered with hexamine and stained for 2 hours in a solution made from $1 \mathrm{~g}$ rose bengal and $5 \mathrm{~g}$ phenol in one litre $4 \%$ formaldehyde in distilled water buffered with borax. The sorting medium was dilute $4 \%$ formaldehyde in distilled water buffered with borax. The specimen illustrated in Fig. 1a was processed by this method; but filtered seawater was used throughout, leading to the precipitation of halite crystals on the test's surface which is why we recommend the use of distilled water, rather than filtered seawater, during the staining and sorting processes. All illustrated specimens are from different samples from the DISCOL (DISturbance and reCOLonization experiment in a manganese nodule area of the South East Pacific) experimental area. It may be argued that the dissolution observed here is due to the experimental area's position close to the $\mathrm{CCD}$. We have, however, observed the same effects of dissolution on shallow, marine specimens that have been processed by the same method and examination of freshly collected material sieved and observed in filtered seawater on shipboard did not reveal any obvious dissolution of calcareous specimens. Adoption of the staining and sorting procedures outlined in this paper and regularly monitoring the $\mathrm{pH}$ of the preservative dramatically reduces the incidence of occurrence of 'dissolved' specimens.

protected and a fume cupboard should be used for weighing this reagent and preparing the buffer solution. To avoid the production of arsenic gas, this reagent should not come into contact with acids. Since cacodylate buffer reacts with $\mathrm{H}_{2} \mathrm{~S}$, the two should not be used in the same solution. Cacodylate solutions should not be poured down the sink, since they can react with drain cleaners and other chemicals to produce toxic arsenic gas' - Hayat, op. cit. 


\section{METHOD 2: FORMALDEHYDE}

\section{Fixation}

Prepare a solution of $2 \%$ formaldehyde from laboratory reagent quality paraformaldehyde by dissolving $2 \mathrm{~g}$ of paraformaldehyde in $100 \mathrm{ml}$ filtered seawater. It is dissolved by heating the mixture in a fume cupboard to $60^{\circ} \mathrm{C}$, stirring constantly and adding drops of $1 \mathrm{M}$ sodium hydroxide (prepared by dissolving $40 \mathrm{~g}$ analytical reagent quality sodium hydroxide pellets in 11 distilled water) until the powder dissolves. Buffer with $0.8 \mathrm{~g}$ analytical reagent quality di-sodium tetraborate (=borax) per $100 \mathrm{ml} 2 \%$ formaldehyde in filtered seawater ( $\mathrm{pH} 9.22$ at $20^{\circ} \mathrm{C}$ ). Borax (pers. observation) is a more satisfactory buffer for formaldehyde than analytical reagent quality hexamine, maintaining hydrogen ion equilibrium for longer time intervals. Add fixative to the marine sediments as above; but the fixative:sediment ratio volume should be 9:1.

The advantages of using paraformaldehyde rather than formalin are that it will contain only unpolymerized formaldehyde, which will penetrate and fix the tissue more easily than that available in commercial preparations, and formalin also contains unwanted stabilizing agents such as methanol and formic acid. In addition, the storage of paraformaldehyde is $100 \%$ safe as it is in the form of a powder.

\section{Preservation}

In this method the fixative also functions as the preservative. The $\mathrm{pH}$ of this solution should be checked every month and the solution changed if its value falls below 8.6. We do not recommend adding more di-sodium tetraborate to maintain alkalinity as this tends to recrystallize on the surfaces of foraminiferal tests (J.R. Haynes, pers. observation). The optimum storage temperature for the formaldehyde solution is between 5 and $15^{\circ} \mathrm{C}$, (Hodgkinson, op. cit.).

\section{Staining \\ See method 1 .}

\section{Sorting}

See method 1

\section{Specific hazards}

Formaldehyde/paraformaldehyde is toxic by inhalation and ingestion. Precise details of its harmful effects should be obtained from the BDH Hazard Data Sheets from Merck Ltd. More seriously, a 'carcinogen can form spontaneously in air when formaldehyde and hydrogen chloride vapours mix. Formaldehyde has also been reported to cause nasal and squamous cell carcinoma in animals exposed to 11$15 \mathrm{ppm}$. About 5ppm is the limit for a brief exposure, while 2ppm is the limit for continued or repeated exposure and is the level at which formaldehyde can just be smelt. The possible carcinogenic effect of formaldehyde in the human respiratory tract is currently a matter of debate (Perera \& Petito, 1982). Some of the pathological effects of exposure to formaldehyde have been reviewed by Loomis (1979)' Hayat, op. cit.

\section{This is DISCOL publication number 29.}

\section{ACKNOWLEDGEMENTS}

This work has arisen from CM's participation in the DISCOL (DISturbance and reCOLonization experiment in a manganese nodule area of the South East Pacific) project and is supported by NERC Grant No. GR3/7309A, which is gratefully acknowledged. We are indebted to $\mathrm{Dr}$ R.E. Jenkins, Research Education and Healthcare Manager of Merck UK for his advice and permission to quote information from Merck's handbooks to Professor M.A. Hayat, Kean College, Union, New Jersey, USA for permission to quote hazard data from his book: Principles and techniques of electron microscopy and to Professors J.R. Haynes and R.C. Whatley, IES, Aberystwyth UK and Professor H. Thiel and Dr. G. Schriever IHF, Hamburg, Germany for their very useful comments and improvements to the manuscript.

\section{Manuscript received June 1992}

Revised manuscript accepted January 1993

\section{REFERENCES}

Batten, D.J. \& Morrison, L. 1983. Methods of palynological preparation for palaeoenvironmental, source potential and organic maturation studies. Norwegian Petroleum Directorate Bulletin, 2, 35-53

BDH Laboratory Supplies Catalogue. Merck Ltd. 1992 (Most recent edition).

BDH Hazard Data Sheets and Addendum. Merck Ltd. 1990, 1991.

Hay, W.W. 1977. Calcareous nannofossils. In: Ramsay, A.T.S. (Ed.), Oceanic micropalaeontology, 1055-1200. Academic Press, London.

Hayat, M.A. 1989. Principles and techniques of electron microscopy: biological applications, $469 \mathrm{pp}$., Macmillan, London

Hodgkinson, R.L. 1991. Microfossil processing: a damage report. Micropaleontology, New York, 37(3), 320-326.

Lewin, J.C. 1961. The dissolution of silica from diatom walls. Geochim. cosmochim. Acta, London, 21, 182-198.

Loomis, T.A. 1979. Formaldehyde toxicity. Arch. Pathol. lab. Med. American Medical Association, Chicago, 103, 321.

Maybury, C., Morrison, L. \& Stewart, V. 1991. Micropalaeontology notebook. The search for a reliable mounting medium for Recent 'live' foraminifera. J. micropalaeontol, $10(1), 16$.

Perera, F. \& Petito, C. 1982. Formaldehyde: a question of cancer policy. Science, N.Y., 216, 1285-1291.

Pfannkuche, O. \& Thiel, H. 1988. Sample Processing. In: Higgins, R.P. \& Thiel, H. (Eds), Introduction to the study of meiofauna, 134145, Smithsonian Institution Press, Washington, D.C., London. 